\title{
Nonlinear excitation of density fluctuations in electron-magnon systems
}

\author{
L. C. M. Miranda \\ Departamento de Fisica, ${ }^{*}$ Universidade Federal de Pernambuco, 50000 Recife, Brazil \\ and Department of Physics, ${ }^{\dagger}$ University of Arizona, Tucson, Arizona 85721
}

(Received 19 September 1974)

\begin{abstract}
A nonlinear mechanism is suggested for exciting electron-magnon density fluctuation by means of a longwavelength radiation field in doped magnetic semiconductors. A. calculation of the threshold field for this phenomenon is presented in the microwave-frequency region.
\end{abstract}

\section{INTRODUCTION}

With the development of high-intensity radiation sources in the microwave, infrared, and optical spectra the study of the nonlinear interaction of electromagnetic radiation with matter stimulated considerable theoretical and experimental interest. One area of interest is the nonlinear coupling of radiation with spin waves ${ }^{1-3}$ in magnetic materials. In these experiments one applies a $\mathrm{rf}$ radiation to pump spin waves, and one is usually interested in the magnon-photon coupling and magnon-phonon coupling. On the other hand, the electron-magnon influence is often neglected. ${ }^{3}$ In the case of magnetic semiconductors, however, the electron-magnon interaction should also be taken into account.

As is well known, the electron-magon interaction can influence considerably the magnon properties in metals and semiconductors. ${ }^{4-6}$ It is therefore natural to expect that external fields changing the spectrum and the occupation number of the electron states will influence the spin-wave properties. For instance, one knows that under the influence of a fairly strong dc electric field the magnon damping in ferromagnetic semiconductors may change considerably in magnitude and even in sign. The modification of the spin-wave damping in a constant electric field has been discussed by several authors. ${ }^{7-9}$ In Ref. 9 it was suggested that one might possibly observe spin-wave amplification in high-mobility magnetic semiconductors, such as doped $\mathrm{CdCr}_{2} \mathrm{Se}_{4}$, in parallel pumping experiments in which the sample is subjected to an additional dc electric field. The extention of the above theory to account for the effect of the rf radiation on the amplification coefficient of the spin waves was later given elsewhere. ${ }^{10}$ If, however, in these experiments one lets the microwave power increase, one would expect plasmons and magnons to be coupled and excited nonlinearly via the electromagnetic field in analogy with the radiation-induced nonlinear plasmon-phonon coupling in doped semiconductors. ${ }^{11,12}$

In this paper we consider a system of interacting electrons and magnons and study, within the random-phase approximation, the excitation of resonant density fluctuations of this system driven by long-wavelength electromagnetic radiation. To see how this excitation is possible, we will simply assume that the radiation field can be represented by an oscillatory homogeneous electric field. In the linear approximation the field will cause the electrons to oscillate, and a magnon of frequency $\omega$ couples to electron density fluctuation at the same frequency, $\omega$. However, under the influence of a strong external field we also obtain nonlinear coupling to electron density fluctuations of frequencies $\omega+n \omega_{0}, n= \pm 1, \pm 2, \cdots ; \omega_{0}$ being the frequency of the oscillating external field. Similarly, the electron density fluctuation couples, in turn, to the magnon at frequency $\omega$ as well as to magnons of frequencies $\omega+n^{\prime} \omega_{0}$. If, for simplicity, we assume that our system supports only two modes, the magnon mode at $\omega$ and a plasma mode at $\omega-\omega_{0}$, we obtain a coupling mechanism in which the plasma mode at $\omega-\omega_{0}$ is driven by the magnon and the field at frequency $\omega_{0}$, while the magnon mode is in turn driven by the plasma mode and the field. Under these conditions the two modes will simultaneously be excited by the energy supplied by the external field. This becomes macroscopically observable when the growth rate determined from the nonlinear mechanism overcomes the rate of loss by collisions in the system.

\section{FORMULATION}

We consider a magnetic semiconductor as composed of two subsystems, namely, that of the localized magnetic moments (say, $d$ electrons of paramagnetic atoms), which in the phenomenological description is characterized by the magnetization, and that of the conduction electrons ( $s$ electrons), responsible for the transport and other phenomena. The electronic band structure of these ferromagnetic semiconductors has not been solved to a reliable extent. ${ }^{13,14}$ Nevertheless, there is evidence of the usual parabolic conduction band. 
The total Hamiltonian for the system will comprise the $s$-electrons part, the exchange-coupled spin part, and an interaction term. Since we are interested in studying the system below the Curie temperature, we shall introduce the magnon variables straightaway. Also, as our main objective is the excitation of density fluctuations, in writing down the interaction term we shall include only the one which to lowest order couples electron density fluctuations to spin waves. ${ }^{15}$ Thus, in the second-quantization formalism, the Hamiltonian is given by ${ }^{15,16}$

$$
H=H_{0}+\frac{e \hbar}{m \omega_{0}} \sum_{\overrightarrow{\mathrm{p}}}\left[\overrightarrow{\mathrm{p}} \cdot \overrightarrow{\mathrm{E}}_{0}(t)\right] c_{\overrightarrow{\mathrm{p}}}^{\dagger} c_{\overrightarrow{\mathrm{p}}},
$$

where

$$
H_{0}=H_{e}+H_{e-e}+H_{m}+H_{e-m},
$$

with

$$
\begin{aligned}
& H_{e}=\sum_{\overrightarrow{\mathrm{p}}} \epsilon_{\overrightarrow{\mathrm{p}}} c_{\mathrm{p}}^{\dagger} c_{\overrightarrow{\mathrm{p}}}, \\
& H_{e-e}=\frac{1}{2} \sum_{\overrightarrow{\mathrm{p}} \overrightarrow{\mathrm{p}}, \overrightarrow{\mathrm{k}}} \phi \overrightarrow{\mathrm{k}} c_{\overrightarrow{\mathrm{p}}+\overrightarrow{\mathrm{k}}}^{\dagger} c_{\overrightarrow{\mathrm{p}}}^{\dagger}-\overrightarrow{\mathrm{k}} c_{\overrightarrow{\mathrm{p}}}, c_{\overrightarrow{\mathrm{p}}}, \\
& H_{m}=\sum_{\overrightarrow{\mathrm{k}}} \hbar \omega_{\overrightarrow{\mathrm{k}}} b_{\overrightarrow{\mathrm{k}}}^{\dagger} b_{\overrightarrow{\mathrm{k}}}, \\
& H_{e-m}=\sum_{\overrightarrow{\mathrm{p}} \overrightarrow{\mathrm{k}}} c_{\overrightarrow{\mathrm{p}}+\overrightarrow{\mathrm{k}}}^{\dagger} c_{\overrightarrow{\mathrm{p}}}\left(v_{\overrightarrow{\mathrm{k}}} b_{\overrightarrow{\mathrm{k}}}+v_{\overrightarrow{\mathrm{k}}}^{\frac{*}{*}} b_{\overrightarrow{\mathrm{k}}}^{\dagger}\right) .
\end{aligned}
$$

Here $c_{\overrightarrow{\mathrm{p}}}, c_{\mathrm{p}}^{\dagger}$ are, repectively, the electron destruction and creation operators, $\epsilon \overrightarrow{\mathrm{p}}=\hbar^{2} \overrightarrow{\mathrm{p}}^{2} / 2 m$ is the kinetic energy of the electron of momentum $\hbar \overrightarrow{\mathrm{p}}, \phi_{\mathrm{k}}=4 \pi e^{2} / v k^{2}$ represents the electron-electron interaction, $b_{k}$, $b \vec{k}_{k}$ are the magnon annihilation and creation operators, respectively, $\hbar \omega_{k}$ is the unrenormälized magnon energy, and

$$
v_{\mathrm{k}}=\left(2 \pi g \mu_{B}^{2} / v\right)(2 N S)^{1 / 2}\left(k_{z} k_{-} / k_{2}\right)
$$

$\left(k_{ \pm}=k_{x} \pm i k_{y}\right)$ represents the electron-magnon interaction. $N$ is the number of localized spins and $v$ is the volume of the crystal. We have taken the quantization axis as the $z$ axis. The second term in Eq. (1) represents the coupling of the electrons with the radiation field where $\overrightarrow{\mathrm{E}}_{0}(t)=\overrightarrow{\mathrm{E}}_{0} \cos \omega_{0} t$ represents its electric field. Also, we did not write the electron spin explicitly since the interaction mechanism considered does not cause spin flip.

Before we proceed, however, it is now in order a few comments about the Hamiltonian, Eqs. (1)(3). A more detailed discussion is given elsewhere. ${ }^{15-18}$ The conduction-electron-localizedmoments interaction comprise, in general, the well-known $s-d$ interaction, ${ }^{17,18}$ the interaction of the electron magnetic moment with the magnetic field of the spin waves ${ }^{16}$ (dipolar interaction), and the interaction of the electron currents with the vector potential created by the oscillations of the magnetization. ${ }^{16}$ This last term can also be seen to be the $d$-spin-s-orbit interaction (i.e., a spinorbit interaction). As the electron-photon and the electron-plasmon interaction do not involve carrier spin flip, one should consider only the electron-magnon terms which do not also flip the carrier spin. Of the mechanisms mentioned above, it can be seen that the only relevant electron-magnon terms are the spin-orbit and the dipolar ones. However, since the spin-orbit vertex depends on $\overrightarrow{\mathrm{k}} \times \overrightarrow{\mathrm{p}},{ }^{16}$ it turns out that it gives a vanishing contribution. Hence, in dealing with the simultaneous excitation of a plasmon and a magnon by the radiation field, one may consider only the dipolar electron-magnon term which is given in Eq. (3d).

In order to determine the coupling of the external field to possible density fluctuations, we use the equation-of-motion method ${ }^{19}$ starting from Eq. (1). We shall assume that for $t=-\infty$ the external field is absent and we have a system of noninteracting electrons and magnons in thermal equilibrium, so that for all $\overrightarrow{\mathrm{k}}$ we have $\left\langle b_{k}\right\rangle_{t=-\infty}=0$. Then we consider that the electron-magnon interaction and the external field are set in operation in an adiabatic manner, and by means of some external source nonequilibrium magnons and density fluctuations with wave vector $\overrightarrow{\mathrm{k}}$ are excited, so that starting from this instant, $\left\langle b_{\vec{k}}(t)\right\rangle \neq 0$ and $\left\langle c_{\vec{p}}(t) c_{\vec{p}}+\vec{k}(t)\right\rangle \neq 0$. It is the subsequent fate of the oscillations which we intend to determine.

Define

$$
F(\overrightarrow{\mathrm{p}}+\overrightarrow{\mathrm{k}}, \overrightarrow{\mathrm{p}}, \overrightarrow{\mathrm{t}})=\left\langle\stackrel{\dagger}{c_{\mathrm{p}}^{\dagger}}(t) c_{\overrightarrow{\mathrm{p}}+\overrightarrow{\mathrm{k}}}(t)\right\rangle .
$$

Here $\langle\cdots\rangle$ means the thermal average. It follows from Eqs. (1), (2), and (4) that

$$
\begin{aligned}
i \hbar \frac{\partial F(\overrightarrow{\mathrm{p}}+\overrightarrow{\mathrm{k}}, \overrightarrow{\mathrm{p}}, \overrightarrow{\mathrm{t}})}{\partial t}= & \left(\epsilon_{\overrightarrow{\mathrm{p}}+\overrightarrow{\mathrm{k}}}-\epsilon_{\overrightarrow{\mathrm{p}}}\right) F(\overrightarrow{\mathrm{p}}+\overrightarrow{\mathrm{k}}, \overrightarrow{\mathrm{p}}, \overrightarrow{\mathrm{t}})+\sum_{\overrightarrow{\mathrm{k}}^{\prime}} \phi_{\overrightarrow{\mathrm{k}}, N_{\overrightarrow{\mathrm{k}}}}(t)\left[F\left(\overrightarrow{\mathrm{p}}+\overrightarrow{\mathrm{k}}-\overrightarrow{\mathrm{k}}^{\prime}, \overrightarrow{\mathrm{p}}, \overrightarrow{\mathrm{t}}\right)-F\left(\overrightarrow{\mathrm{p}}+\overrightarrow{\mathrm{k}}, \overrightarrow{\mathrm{p}}+\overrightarrow{\mathrm{k}}^{\prime}, t\right)\right] \\
& +\sum_{\overrightarrow{\mathrm{k}}^{\prime}}\left[v_{\overrightarrow{\mathrm{k}}},\left\langle b_{\overrightarrow{\mathrm{k}}^{\prime}}(t)\right\rangle+v_{\mathrm{k}^{\prime}}^{*}\left\langle b_{-\overrightarrow{\mathrm{k}}^{\prime}}^{\dagger}(t)\right\rangle\right]\left[F\left(\overrightarrow{\mathrm{p}}+\overrightarrow{\mathrm{k}}-\overrightarrow{\mathrm{k}}^{\prime}, \overrightarrow{\mathrm{p}}, t\right)-F\left(\overrightarrow{\mathrm{p}}+\overrightarrow{\mathrm{k}}, \overrightarrow{\mathrm{p}}+\overrightarrow{\mathrm{k}}^{\prime}, t\right)\right] \\
& +\frac{e \hbar}{m \omega_{0}} \overrightarrow{\mathrm{k}} \cdot \overrightarrow{\mathrm{E}}_{0} \cos \omega_{0} t F(\overrightarrow{\mathrm{p}}+\overrightarrow{\mathrm{k}}, \overrightarrow{\mathrm{p}}, \vec{t}),
\end{aligned}
$$


where

$$
N_{\overrightarrow{\mathrm{k}}}(t)=\sum_{\overrightarrow{\mathrm{p}}} F(\overrightarrow{\mathrm{p}}+\overrightarrow{\mathrm{k}}, \overrightarrow{\mathrm{p}}, t) .
$$

Similarly the equation of motion for the magnon coordinates is

$$
\frac{\partial^{2}}{\partial t^{2}}\left\langle b_{\vec{k}}(t)\right\rangle+\omega \vec{k} \frac{2}{k}\left\langle b_{\vec{k}}(t)\right\rangle=-\frac{\omega \overrightarrow{\mathrm{k}} v_{\overrightarrow{\mathrm{k}}}^{*}}{\hbar} N_{\overrightarrow{\mathrm{k}}}(t) .
$$

In arriving at Eq. (5), the electron-electron correlations (collisions) have been neglected; however, the self-consistent field of the electrons has been taken into account. Also, we considered the electron-magnon interaction only within the framework of the random phase approximation.

To solve for $F(\overrightarrow{\mathrm{p}}+\overrightarrow{\mathrm{k}}, \overrightarrow{\mathrm{p}}, t)$ we use perturbation theory in the electron-magnon coupling but retain $E_{0}$ to all orders. We write

$$
F(\overrightarrow{\mathrm{p}}+\overrightarrow{\mathrm{k}}, \overrightarrow{\mathrm{p}}, t)=f_{0}(\overrightarrow{\mathrm{p}}) \delta \overrightarrow{\mathrm{k}}, 0+f(\overrightarrow{\mathrm{p}}+\overrightarrow{\mathrm{k}}, \overrightarrow{\mathrm{p}}, t),
$$

where $f_{0}(\overrightarrow{\mathrm{p}})$, the zeroth-order solution, is the Fermi distribution function and is time independent. Similar procedure has been used elsewhere. ${ }^{20}$ Substituting Eq. (8) into Eqs. (5) and (7), to first order in the electron-magnon coupling, we get the closed set of equations

$$
\begin{aligned}
& \begin{aligned}
i \hbar \frac{\partial f(\overrightarrow{\mathrm{p}}+\overrightarrow{\mathrm{k}}, \overrightarrow{\mathrm{p}}, t)}{\partial t}= & \left(\epsilon_{\overrightarrow{\mathrm{p}}+\overrightarrow{\mathrm{k}}}-\epsilon_{\overrightarrow{\mathrm{p}}}\right) f(\overrightarrow{\mathrm{p}}+\overrightarrow{\mathrm{k}}, \overrightarrow{\mathrm{p}}, t)+\phi \overrightarrow{\mathrm{k}} n_{\overrightarrow{\mathrm{k}}}(t)\left[f_{0}(\overrightarrow{\mathrm{p}})-f_{0}(\overrightarrow{\mathrm{p}}+\overrightarrow{\mathrm{k}})\right] \\
& +\left[v_{\overrightarrow{\mathrm{k}}}\left\langle b_{\overrightarrow{\mathrm{k}}}(t)\right\rangle+v_{\overrightarrow{\mathrm{k}}}^{*}\left\langle b_{-\overrightarrow{\mathrm{k}}}^{\dagger}(t)\right\rangle\right]\left[f_{0}(\overrightarrow{\mathrm{p}})-f_{0}(\overrightarrow{\mathrm{p}}+\overrightarrow{\mathrm{k}})\right]+\frac{e \hbar}{m \omega_{0}}\left(\overrightarrow{\mathrm{k}} \cdot \overrightarrow{\mathrm{E}}_{0}\right) \cos \omega_{0} t f(\overrightarrow{\mathrm{p}}+\overrightarrow{\mathrm{k}}, \overrightarrow{\mathrm{p}}, t),
\end{aligned} \\
& i \frac{\partial^{2}}{\partial t^{2}}\left\langle b_{\overrightarrow{\mathrm{k}}}(t)\right\rangle+\omega_{\overrightarrow{\mathrm{k}}}^{2}\left\langle b_{\overrightarrow{\mathrm{k}}}(t)\right\rangle=-\frac{\omega_{\overrightarrow{\mathrm{k}}} v_{\mathrm{k}}^{*}}{\hbar} n_{\overrightarrow{\mathrm{k}}}(t), \\
& n_{\overrightarrow{\mathrm{k}}}(t)=\sum_{\overrightarrow{\mathrm{p}}} f(\overrightarrow{\mathrm{p}}+\overrightarrow{\mathrm{k}}, \overrightarrow{\mathrm{p}}, t) .
\end{aligned}
$$

In order to solve Eqs. (9)-(11) we first use a transformation which eliminates the field from our equations. Defining

$$
f(\overrightarrow{\mathrm{p}}+\overrightarrow{\mathrm{k}}, \overrightarrow{\mathrm{p}}, t)=\tilde{\mathrm{f}}(\overrightarrow{\mathrm{p}}+\overrightarrow{\mathrm{k}}, \overrightarrow{\mathrm{p}}, t) e^{-i \lambda \sin \omega_{0} t}
$$

with

$$
\lambda=\frac{e}{m \omega_{0}^{2}} \overrightarrow{\mathrm{k}} \cdot \overrightarrow{\mathrm{E}}_{0},
$$

we obtain the following kinetic equation for $\tilde{f}$ :

$$
\begin{aligned}
\left(i \hbar \frac{\partial}{\partial t}\right. & \left.+\epsilon_{\overrightarrow{\mathrm{p}}}-\epsilon_{\overrightarrow{\mathrm{p}}+\overrightarrow{\mathrm{k}}}\right) \tilde{f}(\overrightarrow{\mathrm{p}}+\overrightarrow{\mathrm{k}}, \overrightarrow{\mathrm{p}}, t)-\phi_{\overrightarrow{\mathrm{k}}}\left[f_{0}(\overrightarrow{\mathrm{p}})-f_{0}(\overrightarrow{\mathrm{p}}+\overrightarrow{\mathrm{k}})\right] \rho_{\overrightarrow{\mathrm{k}}}(t) \\
& =e^{i \lambda \sin \omega_{0} t}\left[v_{\overrightarrow{\mathrm{k}}}\left\langle b_{\overrightarrow{\mathrm{k}}}(t)\right\rangle+v_{\mathrm{k}}^{*}\left\langle b_{-\overrightarrow{\mathrm{k}}}^{\dagger}(t)\right\rangle\right]\left[f_{0}(\overrightarrow{\mathrm{p}})-f_{0}(\overrightarrow{\mathrm{p}}+\overrightarrow{\mathrm{k}})\right] .
\end{aligned}
$$

Similarly, the magnon variables obey

$$
i \frac{\partial^{2}}{\partial t^{2}}\left\langle b_{\vec{k}}(t)\right\rangle+\omega_{\mathrm{k}}^{2}\left\langle b_{\vec{k}}(t)\right\rangle=-\frac{\omega_{\vec{k}} v_{\mathrm{k}}^{*}}{\hbar} \rho \vec{k}_{\mathrm{k}}(t) e^{-i \lambda \sin \omega_{0} t},
$$

where

$$
\rho \overrightarrow{\mathrm{k}}(t)=\sum_{\overrightarrow{\mathrm{p}}} \tilde{f}(\overrightarrow{\mathrm{p}}+\overrightarrow{\mathrm{k}}, \overrightarrow{\mathrm{p}}, t) .
$$

By looking at Eq. (14) the phy sical mechanism for density excitation via the field is now clear. It is the difference between the oscillatory motions of the electrons and the magnons in applied fields which couple electron-density fluctuations at frequency $\omega$ with magnons at frequencies $\omega+s \omega_{0}$, and conversely.

The solution of Eqs. (14)-(16) is given in terms of the Fourier spectra of $\tilde{f}$, and where

$$
\tilde{f}(t)=\tilde{f}(\omega) e^{-i(\omega+i \eta) t} \text {, etc. }
$$

We define the following quantities:

$$
P \overrightarrow{\mathrm{k}}(\omega)=\sum_{\overrightarrow{\mathrm{p}}} \frac{f_{0}(\overrightarrow{\mathrm{p}}+\overrightarrow{\mathrm{k}})-f_{0}(\overrightarrow{\mathrm{p}})}{\epsilon_{\overrightarrow{\mathrm{p}}}+\overrightarrow{\mathrm{k}}-\epsilon_{\overrightarrow{\mathrm{p}}}-\hbar \omega},
$$




$$
\begin{aligned}
& \epsilon_{k}(\omega)=1-\phi_{\overrightarrow{\mathrm{k}}} P_{\overrightarrow{\mathrm{k}}}(\omega), \\
& D_{\overrightarrow{\mathrm{k}}}(\omega)=\frac{1}{\omega^{2}-\omega_{\overrightarrow{\mathrm{k}}}^{2}} .
\end{aligned}
$$

Here $P_{\vec{k}}(\omega)$ is the electronic polarizability, $\epsilon_{\vec{k}}(\omega)$ is the electronic dielectric function, and $D_{\vec{k}}(\omega)$ is the magnon propagator. Solving for $\rho_{\vec{k}}(\omega)$ in Eq. (14), we obtain

$$
\begin{aligned}
\epsilon_{\overrightarrow{\mathrm{k}}}(\omega) \rho_{\overrightarrow{\mathrm{k}}}(\omega)= & P_{\overrightarrow{\mathrm{k}}}(\omega) \sum_{n} J_{n}(\lambda) \\
& \times\left[v_{\overrightarrow{\mathrm{k}}} B_{\overrightarrow{\mathrm{k}}}\left(\omega-n \omega_{0}\right)+v_{\overrightarrow{\mathrm{k}}}^{*} B_{-\overrightarrow{\mathrm{k}}}^{\dagger}\left(\omega-n \omega_{0}\right)\right] .
\end{aligned}
$$

Similarly, from Eq. (15) we get

$$
B_{\overrightarrow{\mathrm{k}}}(\omega)=\frac{\omega_{k} v_{k}^{*}}{\bar{\hbar}} D_{\overrightarrow{\mathrm{k}}}(\omega) \sum_{n} J_{n}(\lambda) \rho_{\overrightarrow{\mathrm{k}}}\left(\omega+\boldsymbol{n} \omega_{0}\right)
$$

Finally, using Eqs. (21) and (22) we obtain the following set of coupled equations:

$$
\begin{aligned}
\epsilon_{\mathrm{k}}\left(\omega+s \omega_{0}\right) \rho_{\mathrm{k}}\left(\omega+s \omega_{0}\right)= & \left|V_{\overrightarrow{\mathrm{k}}}\right|^{2} p_{\overrightarrow{\mathrm{k}}}\left(\omega+s \omega_{0}\right) \\
& \quad \times \sum_{n, l} J_{n-s}(\lambda) J_{l-n}(\lambda) \\
& \quad \times D_{\overrightarrow{\mathrm{k}}}\left(\omega+n \omega_{0}\right) \rho_{\overrightarrow{\mathrm{k}}}\left(\omega+l \omega_{0}\right),
\end{aligned}
$$

$$
\left|V_{\overrightarrow{\mathrm{k}}}\right|^{2}=2 \omega_{\overrightarrow{\mathrm{k}}}\left|v_{\overrightarrow{\mathrm{k}}}\right|^{2} / \hbar
$$

which determine the eigenmodes and possible growth rates for the electron-magnon system driven by the external oscillating field. In the limit $\overrightarrow{\mathrm{E}}_{0}=0$ (i. e., $\lambda=0$ ), only the $s=0$ component is relevant, and we obtain the dispersion relation for the oscillations of the electron-magnon system in the absence of the external field.

\section{NONLINEAR DISPERSION RELATION}

Consider now the system in the two-mode approximation. Here we discuss the situation in which the external field $E_{0}$ excites the two linear modes, the plasmon and magnon. Let us solve Eq. (23) for the case that $\omega$ and $\omega-\omega_{0}$ are, respectively, in the vicinity of the plasma and magnon frequencies. We then obtain the coupled equations :

$$
\begin{aligned}
& \epsilon_{\overrightarrow{\mathrm{k}}}(\omega) \rho_{\overrightarrow{\mathrm{k}}}(\omega)=\left|V_{\overrightarrow{\mathrm{k}}}\right|^{2} P_{\overrightarrow{\mathrm{k}}}(\omega) \sum_{n=0,-1} \sum_{l=0,-1} J_{n}(\lambda) J_{l-n}(\lambda) D_{\overrightarrow{\mathrm{k}}}\left(\omega+n \omega_{0}\right) \rho_{\mathrm{k}}\left(\omega+l \omega_{0}\right), \\
& \epsilon_{\overrightarrow{\mathrm{k}}}\left(\omega-\omega_{0}\right) \rho_{\overrightarrow{\mathrm{k}}}\left(\omega-\omega_{0}\right)=\left|V_{\overrightarrow{\mathrm{k}}}\right|^{2} P_{\overrightarrow{\mathrm{k}}}\left(\omega-\omega_{0}\right) \sum_{n=0,-1} \sum_{l=0,-1} J_{n+1}(\lambda) J_{l-n}(\lambda) D_{\overrightarrow{\mathrm{k}}}\left(\omega+n \omega_{0}\right) \rho_{\overrightarrow{\mathrm{k}}}\left(\omega+l \omega_{0}\right) .
\end{aligned}
$$

The solution of these coupled equations for nonvanishing density fluctuations $\rho_{\vec{k}}(\omega)$ and $\rho_{\vec{k}}\left(\omega-\omega_{0}\right)$ is given by the dispersion relation

$$
\begin{aligned}
& \begin{aligned}
\left\{\epsilon_{\mathrm{k}}(\omega)-\left[J_{0}^{2}(\lambda)+J_{1}^{2}(\lambda)\right] \beta_{k}^{2}\left[1-\epsilon_{\mathrm{k}}(\omega)\right] D_{\mathrm{k}}(\omega)\right\} & \left\{\epsilon_{k}\left(\omega-\omega_{0}\right)-\left[J_{0}^{2}(\lambda)+J_{1}^{2}(\lambda)\right] \beta_{k}^{2}\left[1-\epsilon_{k}\left(\omega-\omega_{0}\right)\right] D_{k}\left(\omega-\omega_{0}\right)\right\} \\
& \times J_{0}^{2}(\lambda) \beta_{k}^{2}\left[\epsilon_{k}\left(\omega-\omega_{0}\right)-\epsilon_{k}(\omega)\right]\left[D_{\overrightarrow{\mathrm{k}}}(\omega)+D_{\mathrm{k}}\left(\omega-\omega_{0}\right)\right]=0,
\end{aligned} \\
& \beta_{k}^{2}=\frac{2 \omega_{k}\left|v_{k}\right|^{2}}{\hbar \phi_{\overrightarrow{\mathrm{k}}}} .
\end{aligned}
$$

For zero external field each of the brackets in Eq. (27) when set equal to zero gives, respectively, the dispersion in relation for a plasmon and a magnon.

In the long-wavelength case we obtain for $E_{0}=0$ the relation

$$
1-\frac{\omega_{p}^{2}}{\omega^{2}}-\frac{\omega_{p}^{2}}{\omega^{4}} 3 v_{T h}^{2} k^{2}-\beta_{k}^{2} \frac{\omega_{p}^{2}}{\omega^{2}} \frac{1}{\omega^{2}-\omega_{\vec{k}}^{2}}=0,
$$

where we have assumed a nondegenerate semiconductor and taken the classical limit for the dielectric function, which holds for microwave regions even for temperatures of $10^{\circ} \mathrm{K}$. Equation (29) determines, respectively, the renormalized plasma frequency $\omega_{1}$, and magnon frequency $\omega_{2}$ given by

$$
\begin{aligned}
\omega_{1,2}^{2}= & \frac{1}{2}\left(\omega_{p}^{2}+\omega_{k}^{2}+3 v_{T h}^{2} k^{2}\right) \\
& \pm \frac{1}{2}\left[\left(\omega_{p}^{2}+3 v_{T h}^{2} k^{2}-\omega_{k}^{2}\right)^{2}+4 \beta_{k}^{2} \omega_{p}^{2}\right]^{1 / 2} .
\end{aligned}
$$

For the case $\beta_{k}^{2}<\omega_{k}^{2}, \omega_{p}^{2}$ and $\beta_{k}^{2}<\left(\omega_{p}-\omega_{k}\right)^{2}$, we obtain the approximate solutions

$$
\begin{aligned}
& \omega_{1}^{2} \approx \omega_{p}^{2}+3 v_{T h}^{2} k^{2}+\beta_{k}^{2} \omega_{p}^{2} /\left(\omega_{p}^{2}-\omega_{k}^{2}\right), \\
& \omega_{2}^{2} \approx \omega_{k}^{2}-\beta_{k}^{2} \omega_{p}^{2} /\left(\omega_{p}^{2}-\omega_{k}^{2}\right) .
\end{aligned}
$$

We next solve Eq. (27) in the regime $k<k_{D}\left(=\omega p / v_{T h}\right)$, which determines the limit for long-wavelength 
phenomena. Using Eqs. (18)-(20) in the long wavelength, Eq.(27) reads, after some algebra,

$$
\begin{aligned}
&\left(\omega^{2}-\omega_{1}^{2}\right)\left(\omega^{2}-\omega_{2}^{2}\right)\left[\left(\omega-\omega_{0}\right)^{2}-\omega_{1}^{2}\right] {\left[\left(\omega-\omega_{0}\right)^{2}-\omega_{2}^{2}\right] } \\
&=J_{1}^{2}(\lambda) \beta_{k}^{2} \omega_{p}^{2}\left(\omega_{1}^{2}-\omega_{2}^{2}\right)^{2},
\end{aligned}
$$

where we have neglected terms of order $k v_{T h} / \omega_{p}$, $k v_{T h} / \omega_{p}$, and already substituted in the right-hand side of Eq. (33) the linear solutions, i. e., $\omega=\omega_{1}$, and $\omega-\omega_{0}=\omega_{2}$. Here $\omega_{1}$ and $\omega_{2}$ are the eigenvalue frequencies of the linear dispersion equation. To get the mode-one growth rate, we substitute $\omega=\omega_{1}$ $+i \gamma$ in Eq. (33). Assuming $\gamma<\omega_{2}, 2 \omega_{1}$ we obtain

$$
|\gamma|=J_{1}(\lambda) \beta_{k} \omega_{p} / 2\left(\omega_{1} \omega_{2}\right)^{1 / 2} \text {. }
$$

In the long wavelength Eq. (34) reduces to

$$
|\gamma| \approx \frac{1}{4} \frac{e k E_{0}}{m\left(\omega_{1}+\omega_{1}\right)} \frac{\beta_{k} \omega_{p}}{\left(\omega_{1} \omega_{2}\right)^{1 / 2}} .
$$

Now as is well known from parametric instability theory ${ }^{21,22}$ that the excitation of the density fluctuations becomes macroscopically obseravable when the growth rate $\gamma$ exceeds the effective collision frequency of the system $\left(\nu_{1} \nu_{2}\right)^{1 / 2}$, where $\nu_{1}$ and $\nu_{2}$ are, respectively, the collision frequency of the two linear modes. This entails in turn that instability occurs when the radiation field strength is larger than a threshold value $\left(E_{0}\right)_{t h}$ given by

$$
\frac{1}{4} \frac{e k\left(E_{0}\right)_{t h}}{m\left(\omega_{1}+\omega_{2}\right)} \frac{\beta_{k} \omega_{p}}{\omega_{1} \omega_{2}}=\left(\omega_{1} \tau_{1}\right)^{-1 / 2}\left(\omega_{2} \tau_{2}\right)^{-1 / 2} .
$$

Therefore, radiation at frequency $\omega_{0}=\omega_{1}+\omega_{2}$ and field $E_{0}>\left(E_{0}\right)_{t h}$ will exhibit an absorption. For a typical sample of $\mathrm{CdCr}_{2} \mathrm{Se}_{4}$ with $\omega_{p}^{2}=10^{22} \mathrm{sec}^{-2}$, $\omega_{k}^{2} \approx 10^{21} \mathrm{sec}^{-2}, \quad v_{\mathrm{Th}} \approx 3 \times 10^{6} \mathrm{~cm} / \mathrm{sec}, \quad m \approx 10^{-29} \mathrm{~g}$, and for $k=10^{5} \mathrm{~cm}^{-1}<k_{D}$ we obtain a threshold field

$$
\left(E_{0}\right)_{t h} \approx 10\left(\omega_{1} \tau_{1}\right)^{-1 / 2}\left(\omega_{2} \tau_{2}\right)^{-1 / 2} \mathrm{~V} / \mathrm{cm}
$$

This relatively large threshold field at microwave frequencies is due to the poor coupling between electron and magnons for small wave numbers. For realistic cases $\omega_{1} \tau_{1} \sim 10$ and $\omega_{2} \tau_{2} \sim 100$, so that $\left(E_{0}\right)_{t h}$ is of the order of $10^{4} \mathrm{~V} / \mathrm{cm}$, which can be obtained using pulse techniques.

\section{CONCLUSIONS}

In this paper we have looked at possible nonlinear excitation of density fluctuations in ferromagnetic semiconductors when performing spin-wave-pumping experiments. It was shown that, as the microwave power increases we could, in principle, expect an absorption line at frequency $\omega_{0}=\omega_{1}+\omega_{2}$, for fields for the order of $10^{4} \mathrm{~V} / \mathrm{cm}$. It is also clear that the additional absorption of the radiation and the excitation of plasmons and magnons is an onoff phenomenon: No excitation is possible below $\left(E_{0}\right)_{t h}$, and strong excitation exists for $E_{0}>\left(E_{0}\right)_{t h}$. As there has been to the author's knowledge no direct experimental studies of the phenomenon discussed in this paper, one would like to suggest spin-wave-pumping experiments in high-power level in order to observe the predictions of this theory. In particular, an experimental determination of $\left(E_{0}\right)_{t h}$ allows us to determine the plasmon lifetime, and hence, the single-electron lifetime, ${ }^{23}$ provided the magnon lifetime is known.

In conclusion, we have calculated the density excitation of electron-magnon system via radiation as a realistic model for magnetic semiconductors and found the threshold field to be of the order of $10^{4} \mathrm{~V} / \mathrm{cm}$ in the microwave region.

\section{ACKNOWLEDGMENTS}

I am indebted to Professor S. M. Rezende and Professor C. B. de Araujo for useful discussions and critical reading of the manuscript.
*Partially supported by CNPg and BNDE (Brazilian Government).

$\dagger$ John Simon Guggenheim Fellow. Present address.

${ }^{1}$ E. Schlömann, J. J. Green, and U. Milano, J. Appl.

Phys. Suppl. 31, 386 (1960).

${ }^{2}$ F. R. Morgenthaler, J. Appl. Phys. Suppl. 31, 95 (1966).

${ }^{3}$ H. Le Gall, Phys. Status Solidi 28, 495 (1968).

${ }^{4}$ R. M. White and R. B. Woosey, Phys. Rev. 196, 908 (1968).

${ }^{5}$ R. B. Woosey and R. M. White, Phys. Rev. B 1,4494 (1970).

${ }^{6} \mathrm{M}$. D. Coutinho, Jr., Ph.D. dissertation (University of São Paulo, São Paulo, 1973) (unpublished).

${ }^{7}$ A. I. Akhiezer, V. G. Baryakhtar, and S. V. Peletminskii, Phys. Lett. $\underline{4} 129$ (1963).
${ }^{8}$ B. Vural, J. Appl. Phys. 37, 1030 (1966).

${ }^{9}$ M. D. Coutinho, Jr., L. C. M. Miranda, and S. M. Rezende, Phys. Status Solidi B 57, 85 (1973).

${ }^{10}$ L. C. M. Miranda, Phys. Status Solidi B 60, 619 (1973).

${ }^{11}$ D. F. DuBois and M. V. Goldmann, Phys. Rev. Lett. 14, 544 (1965).

${ }^{12}$ R. A. Stern and N. Tzoar, Phys. Rev. Lett. 17, 903 (1966).

${ }^{13}$ S. J. Cho, Phys. Rev. 157, 632 (1969).

${ }^{14} \mathrm{~T}$. Kasuya and A. Yanase, Rev. Mod. Phys. $\underline{40}, 684$ (1968).

${ }^{15}$ S. Coutinho and L. C. M. Miranda, Rev. Brasil. Fis. (to be published).

${ }^{16} \mathrm{E}$. A. Turov, in Ferromagnetic Resonance, edited by S. V. Vonsovskii (Pergamon, Oxford, 1966), p. 144. 
${ }^{17}$ S. V. Vonsovskii, Zh. Eksp. Teor. Fiz. 16, 980 (1946).

${ }^{18} \mathrm{~T}$. Kasuya, Progr. Theor. Phys. 16, 45 (1956).

${ }^{19} \mathrm{D}$. Pines, in The Many-Body Problem, edited by

D. Pines (Benjamin, New York, 1962).

${ }^{20}$ É. M. Éphstein, Fiz. Tverd. Tela 11, 2894 (1969) [ Sov. Phys.-Solid State 11, 2327 (1970)] .
${ }^{21}$ M. V. Goldmann, Ann. Phys. (N. Y.) 39, 95 (1966).

${ }^{22}$ M. V. Goldmann, in Nonlinear Effects in Plasmas, edited by G. Kalman and M. Feix (Gordon and Breach, New York, 1969), p. 335.

${ }^{23}$ H. Fröhlich and H. Pelzer, Proc. Phys. Soc. Lond. A 68,525 (1955). 